\title{
Ultrasound-guided central venous catheter placement through the axillary vein in cardiac critical care patients: safety and feasibility of a novel technique in a prospective observational study
}

\author{
Andrea FARINA 1 *, Guido COPPOLA 2 , Giorgio BASSANELLI 1, \\ Alfredo BIANCHI 1, Laura LENATTI 1, Luca A. FERRI 1, Biagio LICCARDO 2, \\ Elena SPINELLI ${ }^{3}$, Stefano SAVONITTO ${ }^{1}$, Tommaso MAURI ${ }^{3}$
}

1Department of Cardiovascular Surgery, Alessandro Manzoni Hospital, Lecco, Italy; 2Division of Cardiology, Monaldi Hospital, Naples, Italy; ${ }^{3}$ Department of Anesthesia, Critical Care and Emergency, Maggiore Polyclinic Hospital, Ca' Granda IRCCS and Foundation, University of Milan, Milan, Italy

*Corresponding author: Andrea Farina, Department of Cardiovascular Surgery, Alessandro Manzoni Hospital, via dell'Eremo 9/11, 23900 Lecco, Italy. E-mail: a.farina@asst-lecco.it

\section{A B S T R A C T}

BACKGROUND: Central venous catheterization is essential for careful administration of fluids and drugs in cardiac critical care patients. The axillary vein might represent an alternative to subclavian and jugular vein accesses, with the advantage of being extra-thoracic, more distal from the pleural space and with more likehood of comfort for the patient. Conventional ultrasound-guided cannulation of the axillary vein is technically demanding and does not guarantee precise visualization of the needle tip.

METHODS: We describe a new in-plane technique with a dedicated bracket support for the needle, giving full tip control and continuous visualization of the tip and vessel, making the maneuver easier and safer. In a prospective observational study we also report the feasibility and safety of the novel procedure in a series of 35 cardiac critical care patients, also receiving non-invasive ventilatory support and/or being fully anti-coagulated.

RESULTS: With the novel technique, we obtained $97 \%$ success with procedural times comparable to other insertion sites and without complications.

CONCLUSIONS: Placement of a central line catheter in the axillary vein using a novel ultrasound-guided bracketassisted technique may be a feasible, safe and rapid alternative to the conventional jugular and subclavian approaches.

(Cite this article as: Farina A, Coppola G, Bassanelli G, Bianchi A, Lenatti L, Ferri LA, et al. Ultrasound-guided central venous catheter placement through the axillary vein in cardiac critical care patients: safety and feasibility of a novel technique in a prospective observational study. Minerva Anestesiol 2020;86:157-64. DOI: 10.23736/S0375-9393.19.13670-X)

KEY WORDS: Axillary vein; Ultrasonography; Central venous catheters; Cannula.

A ccess to the central venous system is of paramount importance in critical care medicine. For cardiac patients, measure of the central venous pressure and oxygen saturation allows careful fluid infusion avoiding overload. The first choice is usually jugular out-of-plane ultrasound (US)-guided cannulation; the subclavian vein is an alternative associated with more morbidity. ${ }^{1-4}$ The axillary vein, originating from the confluence of the brachial and basilica veins at the inferior margin of pectoral muscle and overriding the first rib continuing as subclavian vein, is an alternative, yet rarely used. It has the advantage of being extra-thoracic and farther from the pleural cavity 


\section{COPYRIGHT $^{\circledR} 2020$ EDIZIONI MINERVA MEDICA}

but lacks precise anatomical landmarks, 5, 6 and only an US-guided approach makes it a viable option. In previous literature, there is some experience of US-guided axillary puncture, but the described procedures include introducer-needle inserted at a steep angle to the chest wall and outof-plane in respect to the ultrasound beam. ${ }^{7-9}$ This way does not guarantee continuous visualization of the true tip of the needle, with some risk of accessing the thorax between the ribs. Moreover, the out-of-plane technique does not allow continuous real-time guidance for all the procedural steps. The less commonly used free-hand in-plane technique can not assure constant visualization of the true needle tip, because even minimal movements of the hand during the procedure can significantly displace the tip and/or the direction of the probe, and adjustments on the longitudinal axis to visualize the trajectory of the needle are technically complex;10-12 an intermediate approach with the same limitations is the oblique axis view + in-plane puncture (OA-IP technique). ${ }^{13}$ Finally, in patients with full anti-coagulation or strong intra-thoracic pressure respiratory swings (both common in cardiac critical care patients) the traditional free-hand approach to the axillary vein may become even more hazardous.

The objective of the present study was to describe a novel US-guided technique for cannulation of the axillary vein, in which a dedicated bracket guarantees real-time visualization of both the vein and the needle tip throughout the whole procedure, with the hypothesis of high first pass success and low complications rate. We also report a series of cardiac critical care patients in whom the procedure was performed to demonstrate feasibility, efficacy and safety of this approach.

\section{Materials and methods}

\section{Patient population}

Eligible participants were all consecutive patients admitted to the Intensive Cardiac Care Unit (ICCU) of Manzoni Hospital in Lecco, Italy, between January 2018 and December 2018 needing central venous cannulation as per clinical indication. They were assessed by one of two attending physicians for axillary approach because internal jugular or subclavian veins were unavailable (e.g. catheters in place), contraindicated (e.g. anticoagulation for subclavian access) or anatomically difficult (e.g. impossibility to interrupt non-invasive ventilation [NIV] or continuous positive airway pressure [CPAP] delivered with the helmet). The operators were experienced in central venous cannulation and US-guidance. As control group to compare procedural times, we used 25 patients with internal jugular approach admitted to the same ICCU during the same period of time. We recorded procedural time (seconds), from local anesthesia to echo confirmation of tip position, and any clinical complication related to central cannulation occurring during ICCU stay. The Ethics Committee of the Manzoni Hospital approved the study and, following the EU Regulation n. 536/2014, 14 informed consent was waived due to the critical conditions of the patients.

Supplementary Digital Material 1 (Supplementary Video 1, 3 minutes, 32MB) shows the whole procedure in a trial patient can be found online as a data supplement to this article.

\section{Preparation}

Patients were kept in neutral position with $15^{\circ}$ Trendelenburg and were monitored using continuous electrocardiography, pulse oximetry and non-invasive blood pressure. A bilateral preprocedural US scanning was performed with a portable Philips CX-50 device and a linear vascular high-resolution 10-MHz transducer (L123 ), moving from the clavicular mid-point laterally in a short-axis projection to assess patency, diameter and depth of the axillary vessel; this was in accordance to Rapid Central Vein Assessment ( $\mathrm{RaCeVA}$ ) protocol which on allowed us to choose the vein side and segment with the best diameter, and to keep an optimal vessel-to-catheter ratio of at least of $3: 1.15$ This preliminary phase was also useful to study relations of the vein with confining structures, in particular the axillary artery, its collateral thoraco-acromial trunk (TAT) which can encroach the vein, the pleural line and, more lateral, the brachial plexus (Figure 1). The cannulation procedure was performed under full sterile conditions including mask, cap, full gown, and gloves, without antibiotic prophylaxis nor sedation, if not otherwise indicated. 


\section{COPYRIGHT $^{\circledR} 2020$ EDIZIONI MINERVA MEDICA}

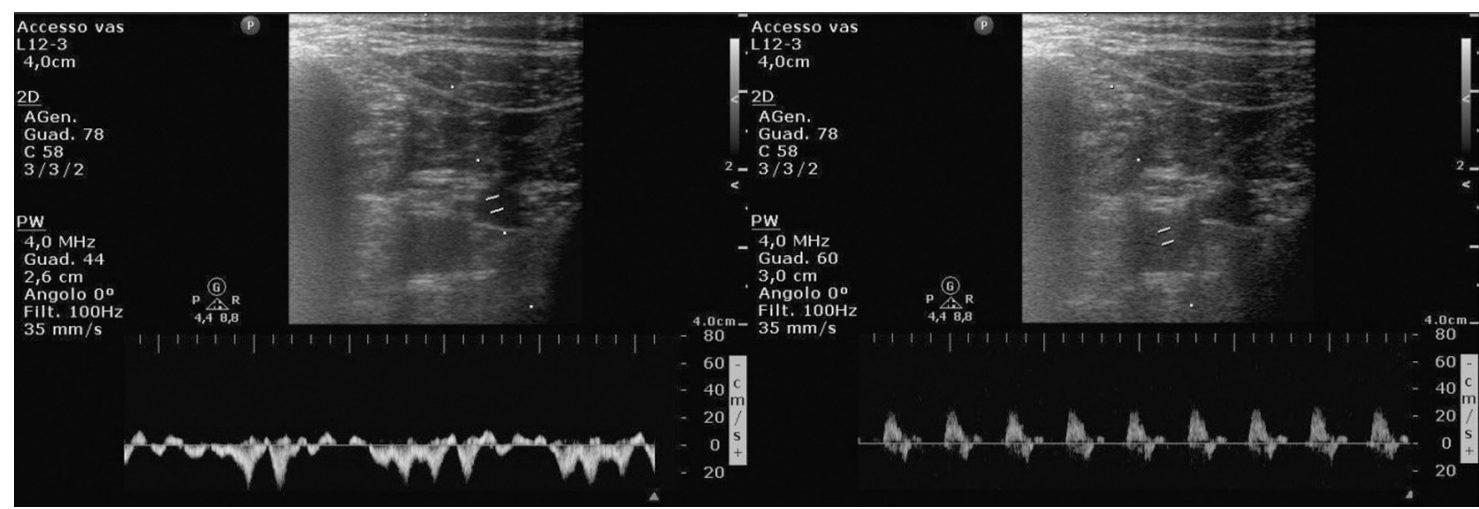

Figure 1.- Short axis view and PW-Doppler profile of axillary vein and artery in the infraclavicular region.

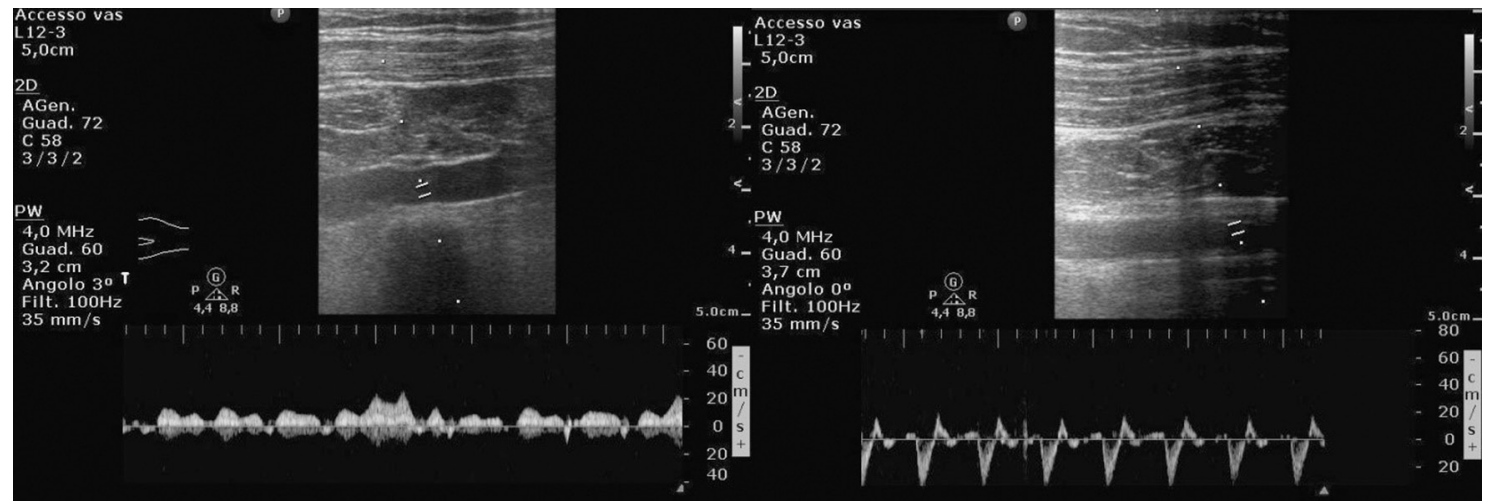

Figure 2.- - Long axis view and PW-Doppler profile of axillary vein and artery in the infraclavicular region.

Skin was cleaned with $2 \%$ alcoholic chlorhexidine (Cloraprep 2\% $6 \mathrm{~mL}$ ) and all the region of interest, head-neck-thorax, was covered with an adhesive holed surgical sheet $(75 \times 90 \mathrm{~cm})$, according to recommendation for maximal barrier precautions. Before sterile wash of the operator, a bracket with three different angles was mounted on the probe to support the needle; the surgical field was set up (disposable suture kit, SURGIKA, Italy); the probe was covered with US gel and wrapped in a sterile sheath, and a 18-gauge 9-cm needle (Cook) was inserted in the disposable support mounted on the covered probe (Ultra-Pro II Needle Guide, CIVCO, IA, USA).

\section{Identification of the vein and in-plane puncture}

Moving to the in-plane projection, the vein course was studied in all its extension; distinction from the artery was recognized by the antero-caudalsuperficial position, the thinner wall, the higher compressibility, the presence of valves and eventually by Doppler and color-Doppler showing a flow phasic with breathing and Valsalva maneuver (Figure 2). The puncture site was chosen laterally in the deltoid-pectoral sulcus, in a segment with sufficient diameter and away from the costal-clavicular stricture, in order to avoid pinch-off risk and to optimize medication. Local anesthesia was administered with $2 \%$ lidocaine. The probe bracket allowed precise advancement of the introducer needle on the plane of the ultrasound beam (Figure 3) until the
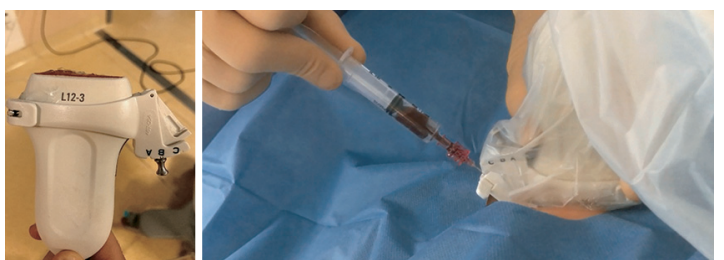

Figure 3.-Bracket mounted on linear probe and in-plane needle advancement. 


\section{COPYRIGHT $^{\odot} 2020$ EDIZIONI MINERVA MEDICA}
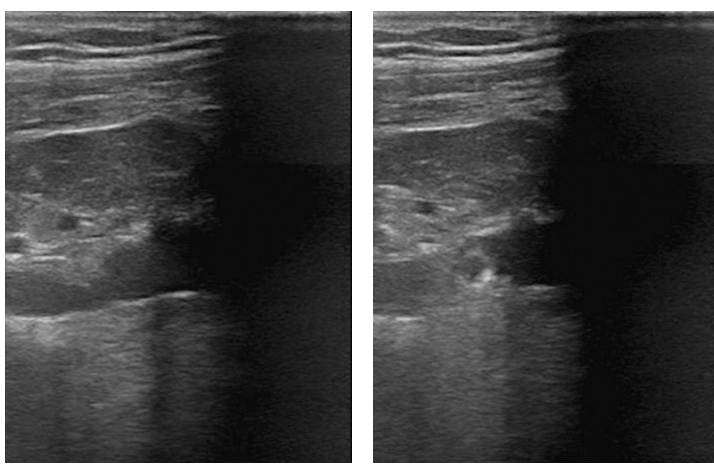

Figure 4.- Needle tip indenting axillary vein wall under direct vision and J-tipped guidewire advancement in axillary vein (on the right clavicular shadowing).

vessel wall appeared indented and blood was freely aspirated in the syringe; then a soft j-tipped guidewire was advanced according to the Seldinger's technique under direct visualization (Figure 4); the probe was then removed, the dilator was advanced with the push-and-twist technique, and finally the catheter or the introducer was inserted. We used any of the following: for central venous access a 7-Fr catheter with a length of $20 \mathrm{~cm}$ for left side or obese patients or $16 \mathrm{~cm}$ for other cases; for pacing, a 5-Fr introducer; for right heart catheterization an 8.5-Fr introducer; for renal replacement therapy a 12-Fr double lumen catheter.

\section{Confirmation of correct positioning}

Trans-thoracic echocardiography, particularly if carried out with contrast enhancement, has proven to be a valid method for the detection of the catheter tip in the distal superior vena cava or in the right atrium and is always available in ICCU. ${ }^{4-16}$ At the end of the procedure we performed a rapid injection of a mixed air-saline solution bolus ( $9 \mathrm{~mL}$ of saline and $1 \mathrm{~mL}$ of air) through the distal lumen of the catheter; in the case of correct positioning a dense US image became visible in the right atrium immediately after injection from sub-costal or apical window using a cardiac probe, whereas in the case of catheter misplacement the opacification would have appeared after a delay (more than two cardiac cycles) (Figure 5). In the latter case, the catheter tip was first searched with linear probe in the ipsilateral jugular vein and then by chest radiography in the contralateral anonymous

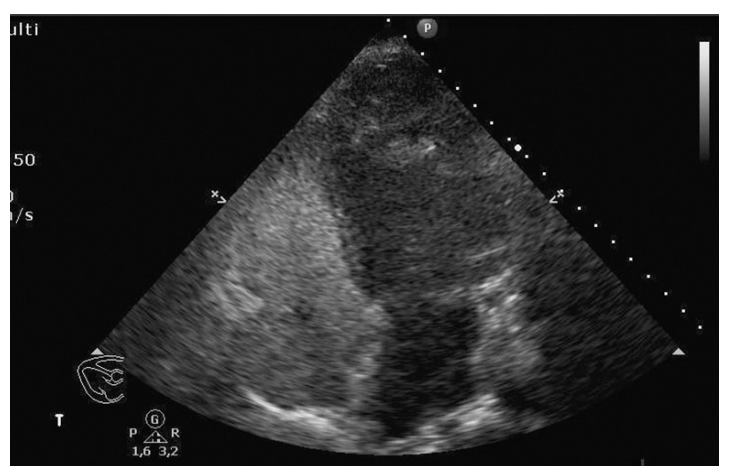

Figure 5.-Bubble test from sub-xiphoid window: immediate right chambers opacification (correct positioning).

vein. ${ }^{17}$ When necessary, US-guided technique was also used for repositioning, allowing the jtip view in the axillary vein during withdrawal of the guidewire and monitoring the correct procedure repositioning during the new advancement (Figure 6). Finally, pneumothorax was excluded with linear probe by appearance of at least one between these signs: lung sliding, lung pulse, Blines. ${ }^{18}$ Chest $\mathrm{X}$-ray was performed anyway to confirm US results.

\section{Statistical analysis}

The study has descriptive purposes and no formal sample size calculation was done. However, a post-hoc calculation of power with 35 patients and no clinical complications or placement failures, allows to exclude a higher than $8 \%$ rate of these events with $95 \%$ confidence level and $80 \%$ power. Continuous variables are presented as means $\pm \mathrm{SD}$ and categorical variables as frequencies $(\%)$. Variables between the axillary and jugular vein cannulation groups were compared by Student's $t$-test (software Microsoft Excel, Edition 2010). A P value of 0.05 was considered statistically significant.

\section{Results}

We performed axillary vein cannulation using the axillary approach in 35 patients: their clinical and procedural characteristics are shown in Table I. Age was $75 \pm 9$ years with BMI $27.3 \pm 5.3$ $\mathrm{Kg} / \mathrm{m}^{2}$. The site of insertion was right in 24 patients and left in 11 patients. We inserted 24 cen- 


\section{COPYRIGHT $^{\odot} 2020$ EDIZIONI MINERVA MEDICA}

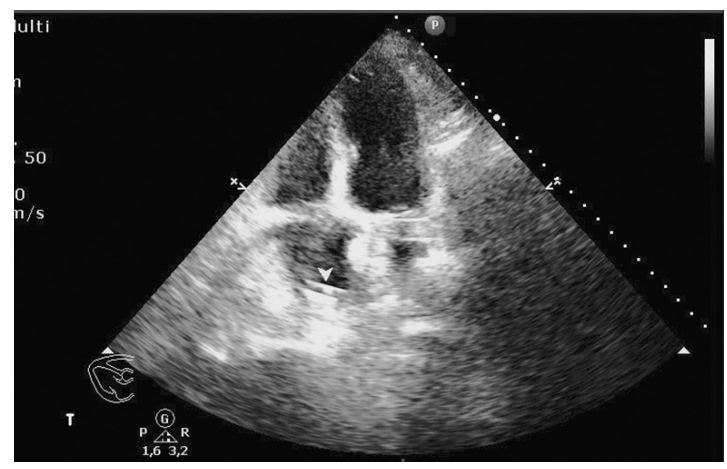

Figure 6.-Correct position of the guidewire in right atrium (arrowhead).

tral venous catheters, six of which $12 \mathrm{Fr}$ dialysis catheters, and 15 introducers (two introducers in the same vessel for patients undergoing permanent pace-maker implantation) with diameter from 5 to $8.5 \mathrm{Fr}$; although this site is notoriously associated with an increased incidence of vein stenosis, which could translate in a problem for future definitive dialysis, temporary CVVH-D was performed from axillary vein in emergency conditions, during anticoagulant therapy, and for the minimal necessary time. A good US window was obtained in all patients, even obese ones, and vein puncture was effective in all at first attempt. Only in one patient, undergoing permanent PM

TABLE I.-Patients and procedural characteristics.

\begin{tabular}{lccc}
\hline Characteristics & $\begin{array}{r}\text { Axillary } \\
(\mathrm{N} .=35)\end{array}$ & $\begin{array}{c}\text { Jugular } \\
(\mathrm{N} .=25)\end{array}$ & $\mathrm{P}$ \\
\hline Age & $74.9 \pm 9.1$ & $72 \pm 8$ & $\mathrm{NS}$ \\
Male gender, N. (\%) & $19(51)$ & $16(64)$ & $\mathrm{NS}$ \\
BMI (kg/m² \pm SD) & $27.3 \pm 5.3$ & $26 \pm 4.8$ & $\mathrm{NS}$ \\
Anticoagulated pts, N. (\%) & $25(67)$ & $17(70)$ & $\mathrm{NS}$ \\
Antiaggregated pts, N. (\%) & $22(59)$ & $15(60)$ & $\mathrm{NS}$ \\
Indications, N. (\%) & & & $\mathrm{NS}$ \\
Fluid/drugs infusion & $18(51)$ & $15(60)$ & \\
CVVHD & $6(17)$ & $3(12)$ & \\
Temporary PM & $2(6)$ & $5(20)$ & \\
$\quad$ Swan Ganz & $5(14)$ & $2(8)$ & \\
Left side access, N. (\%) & $11(31)$ & $3(12)$ & \\
Right side access, N. (\%) & $24(69)$ & $22(88)$ & \\
Procedural time, min \pm DS & $9.3 \pm 2.2$ & $8.5 \pm 1.1$ & \\
$>1$ pucture for access, N. & 0 & 0 & \\
Wire repositioning, N. (\%) & $2(5)$ & 0 & \\
Catheter permanence, days & $5.3 \pm 4.6$ & $3 \pm 1.2$ & \\
\hline
\end{tabular}

CVVHD: continuous venous-venous hemodialysis; PM: pacemaker; PM/ICD: pace maker-internal cardioverter defibrillator; BMI: Body Mass Index; SD: standard deviation. implantation, from the left side advancement of the guide appeared impossible, despite correct puncture of the vessel, and fluoroscopic positioning showed an anatomical variant with kinking of the anonymous vein. In two other patients, there was a malposition from the right axillary vein with the tip ending up in the ipsilateral jugular vein and inducing a transient otalgia; this was detected in one case by US online assessment during the maneuver and in the second case by delayed right atrium opacification after bubble test.In all cases, repositioning was easily performed without new puncture by pulling the j-tip of the wire in axillary vein, under US monitoring, and advancing it in the correct position through right turn of the $\mathrm{J}$ tip and concomitant right arm abduction.

Procedural time, defined as the time elapsed from local anesthesia to confirmation of the correct positioning by means of US, was $9.3 \pm 2.2$ minutes for the axillary approach and it did not differ from control patients with jugular access (Table I).

Of note, $67 \%$ of the patients were on full anticoagulation with heparin or oral anticoagulant (warfarin or dabigatran) and did not have hemorrhagic complications; antiaggregant therapy was present in 22 patients (59\%), with aspirin in all and a second drug (clopidogrel or ticagrelor) in 12 (34\%). One procedure was done during administration of full dose systemic thrombolysis during massive pulmonary embolism and was uneventful.

There were no complications in term of hemothorax, pneumothorax, hematoma, local infection, catheter-related sepsis (Table II). Finally,

TABLE II.-Safety and efficacy endopoints.

\begin{tabular}{lcc}
\hline Characteristics & $\begin{array}{c}\text { Axillary } \\
(\mathrm{N} .=35)\end{array}$ & $\begin{array}{c}\text { Jugular } \\
(\mathrm{N} .=25)\end{array}$ \\
\hline Failure, N. (\%) & $1(3)$ & $0(0)$ \\
Guide-wire malposition, N. (\%) & $2(6)$ & $1(4)$ \\
Hematoma, N. & 0 & 0 \\
Pneumothorax, N. & 0 & 0 \\
Hemothorax, N. & 0 & 0 \\
Local infections, N. & 0 & 0 \\
Catheter-related sepsis, N. & 0 & 0 \\
Brachial plexus injury, N. & 0 & 0 \\
Concordance Echo-Xray control, \% & 100 & NA \\
\hline
\end{tabular}




\section{COPYRIGHT $^{\circledR} 2020$ EDIZIONI MINERVA MEDICA}

the concordance between US bubble-test and chest X-ray for confirmation of correct position was 100 percent.

\section{Discussion}

We report a new US-guided bracket-assisted technique for central venous catheter placement through the axillary vein. We also assessed feasibility and safety of the novel technique in 35 cardiac critical care patients. Finally, comparison with a series of patients receiving jugular catheterization showed comparable results.

Central venous catheterization is an important skill in emergency and critical care medicine. In the cardiac critical care patients it has several indications: infusion of irritants or potent vasoactive drugs, lack of valid peripheral venous access, invasive hemodynamic monitoring for careful infusion therapy, temporary pacing, continuous renal replacement therapy. ${ }^{19,20}$ The traditional access sites are femoral, jugular and subclavian, each of them with both some advantages and disadvantages. The femoral vein is far from the thoracic structures and vital organs, and is useful during cardiac arrest and percutaneous coronary interventions because its cannulation does not interfere with cardiac massage, tracheal intubation or angiographic procedures. The femoral access is the first choice during severe hypothermia because it avoids dangerous intracardiac manipulation of the wire and the risk of precipitating ventricular fibrillation; in all other scenarios it is the last choice because it is associated with an increase in thrombotic and infective complications and limits patient mobilization. Moreover, it does not allow measure of central venous pressure, being the tip of the catheter in the abdomen rather than in the thorax. The subclavian vein is characterized by fixed anatomical landmarks and ligaments that keep it open and make it easily accessible also during shock, but it is an intra-thoracic and uncompressible vessel with the inherent risk of pneumothorax and hemothorax, particularly in the frequent-cases of anti-coagulated cardiac patient. ${ }^{21}$ Moreover, clavicular acoustic shadowing makes it unfavorable for an US approach. Anyway, it is still often used because of a low risk of thrombotic and infec- tive complications and rapidity of cannulation. ${ }^{22}$ Nowadays, the jugular vein is the first recommended site because it is easily suitable for an US-guided cannulation increasing the likelihood of first passage success and reduces the risk of arterial and pleural puncture. Moreover, it allows a briefest and straightest path to the right atrium and is not close to the thoracic duct. However, the jugular vein might be difficult to access in patients with high BMI, short neck or receiving non-invasive ventilation. The axillary-vein approach is still underused because of technically difficulties in echo-guided approach, also with the recently described in-plane approach. ${ }^{23}$

We report a case series with a new technique for cannulation of the axillary vein. We hypothesized that a standardized, easy and safe approach to this cannulation site could become a valid alternative to the jugular vein access, a real alternative due to it being more easily accessible. The axillary vein is not commonly used in emergency and critical care medicine due to the lack of anatomical landmarks and relative complexity in free-hand US-guidance. The previously reported US-assisted out-of-plane puncture does not guarantee real time control of needle position, and also an in-plane puncture has the risk of misalignment between needle tip and ultrasound beam, requiring remarkable manual ability. As compared to previous reports, in the present study, we tried to overcome such limitations by using a dedicated bracket for in-plane approach with firm needle-probe alignment and constant visualization of the needle tip. In our experience, the use of a dedicated bracket to support the maneuver has several advantages: first, it makes the puncture easier to perform, and this is demonstrated in our series from a procedural time comparable with standard jugular cannulation despite years of experience $v s$. no experience with this new approach. Secondly, it makes the position of the needle stable in respect to the probe and allows handling of the guidewire under direct vision. Thirdly, but perhaps most important, this technique gives absolute certainty of real time monitoring of the true needle tip, avoiding puncture of other important structures like the axillary artery, the pleural cavity, and the brachial plexus. Real time echo-guidance allows monitoring of 


\section{COPYRIGHT $^{\circledR} 2020$ EDIZIONI MINERVA MEDICA}

the guidewire path and to promptly redirect it in case of ipsilateral jugular displacement.

The series of patients reported herein demonstrates the feasibility and safety of echo-guided axillary vein puncture with $97 \%$ correct catheter positioning and absence of major complications, even in high risk settings, such as anticoagulated patients and non-invasive ventilation support. Procedural times resulted comparable to the standard jugular approach. Apart from being an alternative as US-guided access, an advantage of this cannulation site might be patient comfort during long-term use of the access and during active mobilization. The ideal exit site is infraclavicular, in the deltoid-pectoral sulcus, an area where dressing is optimal and long-term complications, like infections and thrombosis, are minimal; this exit site is also distant from ventilatory support and tracheostomy. 24

The present study was aimed at assessing the feasibility of the new method, specifically in patients deemed at high-risk of, or unsuitable for, alternative approaches to central vein catheterization, as detailed in the Methods. We would suggest the type of patient appropriate for the axillary approach: presence of multiple central catheters, powerful anti-thrombotic therapy, dependence on non-invasive respiratory support, presence of tracheostomy, high risk of infective complications.

A Health Technology Assessment analysis goes beyond the scope of our study and should include an analysis of the cost of the clinical complications caused/avoided by the present $v s$. alternative methods.

\section{Limitations of the study}

Limitations are the limited sample size, the single center experience, and the non-randomized comparison with the jugular access group; all of these features limit generalisability to others setting and centers.

\section{Conclusions}

In conclusion, the new US-guided bracketassisted axillary vein access for positioning of central venous catheters might represent a safe alternative to more traditional approaches with specific advantages in cardiac critical care patients. More experience also in other subgroups of critical care patients is needed to confirm this preliminary data, especially on the safety of the technique.

\section{What is known}

- Echo-guided central venous catheterization is the standard of care.

- Axillary vein is an alternative to jugular and subclavian vein.

\section{What is new}

- In-plane echo-guided axillary vein cannulation can be assisted by a dedicated bracket for the needle.

- Axillary vein cannulation is effective and rapid also in emergency.

- Axillary vein cannulation is safe also during anti-thrombotic therapy and non-invasive ventilation.

\section{References}

1. Leung J, Duffy M, Finckh A. Real-time ultrasonographically-guided internal jugular vein catheterization in the emergency department increases success rates and reduces complications: a randomized, prospective study. Ann Emerg Med 2006;48:540-7.

2. Graham AS, Ozment C, Tegtmeyer K, Lai S, Braner DA. Videos in clinical medicine. Central venous catheterization. N Engl J Med 2007;356:e21.

3. Troianos CA, Hartman GS, Glas KE, Skubas NJ, Eberhardt RT, Walker JD, et al.; Councils on Intraoperative Echocardiography and Vascular Ultrasound of the American Society of Echocardiography. Guidelines for performing ultrasound guided vascular cannulation: recommendations of the American Society of Echocardiography and the Society of Cardiovascular Anesthesiologists. J Am Soc Echocardiogr 2011;24:1291-318.

4. Lamperti M, Bodenham AR, Pittiruti M, Blaivas M, Augoustides JG, Elbarbary M, et al. International evidencebased recommendations on ultrasound-guided vascular access. Intensive Care Med 2012;38:1105-17.

5. Nickalls RW. A new percutaneous infraclavicular approach to the axillary vein. Anaesthesia 1987;42:151-4.

6. Taylor BL, Yellowlees I. Central venous cannulation using the infraclavicular axillary vein. Anesthesiology 1990;72:55-8.

7. Galloway S, Bodenham A. Ultrasound imaging of the axillary vein - anatomical basis for central venous access. Br J Anaesth 2003;90:589-95. 


\section{COPYRIGHT $^{\circledR} 2020$ EDIZIONI MINERVA MEDICA}

8. Sharma A, Bodenham AR, Mallick A. Ultrasound-guided infraclavicular axillary vein cannulation for central venous access. Br J Anaesth 2004;93:188-92.

9. O’Leary R, Ahmed SM, McLure H, Oram J, Mallick A, Bhambra B, et al. Ultrasound-guided infraclavicular axillary vein cannulation: a useful alternative to the internal jugular vein. Br J Anaesth 2012;109:762-8.

10. Fragou M, Gravvanis A, Dimitriou V, Papalois A, Kouraklis G, Karabinis A, et al. Real-time ultrasound-guided subclavian vein cannulation versus the landmark method in critical care patients: a prospective randomized study. Crit Care Med 2011;39:1607-12.

11. Bodenham AR. Ultrasound-guided subclavian vein catheterization: beyond just the jugular vein. Crit Care Med 2011;39:1819-20.

12. Sommerkamp SK, Romaniuk VM, Witting MD, Ford DR, Allison MG, Euerle BD. A comparison of longitudinal and transverse approaches to ultrasound-guided axillary vein cannulation. Am J Emerg Med 2013;31:478-81.

13. Brescia F, Biasucci DG, Fabiani F, Favarato M, Costa F, Longo $\mathrm{F}$, et al. A novel ultrasound-guided approach to the axillary vein: oblique-axis view combined with in-plane puncture. J Vasc Access 2019;20:763-8.

14. Regulation 536/2014 of the European Parliament and of the Council of 16 April 2014 on clinical trials on medicinal products for human use, and repealing Directive 2001/20/EC of the European Parliament and of the Council of 4 April 2001 on the approximation of the laws, regulations and administrative provisions of the Member States relating to the implementation of good clinical practice in the conduct of clinical trials on medicinal products for human use. Off J Eur Union 2014. L158/1-L158/58.21

15. Pittiruti M. Ultrasound guided central vascular access in neonates, infants and children. Curr Drug Targets 2012;13:961-9.

16. Vezzani A, Brusasco C, Palermo S, Launo C, Mergoni M,
Corradi F. Ultrasound localization of central vein catheter and detection of postprocedural pneumothorax: an alternative to chest radiography. Crit Care Med 2010;38:533-8.

17. Wen M, Stock K, Heemann U, Aussieker M, Küchle C. Agitated saline bubble-enhanced transthoracic echocardiography: a novel method to visualize the position of central venous catheter. Crit Care Med 2014;42:e231-3.

18. Lichtenstein DA, Mezière G, Lascols $N$, Biderman $P$, Courret JP, Gepner A, et al. Ultrasound diagnosis of occult pneumothorax. Crit Care Med 2005;33:1231-8.

19. Rupp SM, Apfelbaum JL, Blitt C, Caplan RA, Connis RT, Domino KB, et al.; American Society of Anesthesiologists Task Force on Central Venous Access. Practice guidelines for central venous access: a report by the American Society of Anesthesiologists Task Force on Central Venous Access. Anesthesiology 2012;116:539-73.

20. Bodenham Chair A, Babu S, Bennett J, Binks R, Fee P, Fox B, et al. Association of Anaesthetists of Great Britain and Ireland: safe vascular access 2016. Anaesthesia 2016;71:573-85.

21. Tercan F, Ozkan U, Oguzkurt L. US-guided placement of central vein catheters in patients with disorders of hemostasis. Eur J Radiol 2008;65:253-6.

22. Parienti JJ, du Cheyron D, Timsit JF, Traoré O, Kalfon $\mathrm{P}$, Mimoz $\mathrm{O}$, et al. Meta-analysis of subclavian insertion and nontunneled central venous catheter-associated infection risk reduction in critically ill adults. Crit Care Med 2012;40:1627-34.

23. Azoulay D, Salloum C, Lahat E, Eshkenazi R, Lim C. A new extra-thoracic, in-plane, longitudinal, real-time, ultrasound-guided access to the axillary vein. Intensive Care Med 2019;45:876-80.

24. Biasucci DG, Pittiruti M, Taddei A, Picconi E, Pizza A, Celentano D, et al. Targeting zero catheter-related bloodstream infections in pediatric intensive care unit: a retrospective matched case-control study. J Vasc Access 2018;19:119-24.

Conflicts of interest.-The authors certify that there is no conflict of interest with any financial organization regarding the material discussed in the manuscript.

Comment in: Biasucci DG. Ultrasound based innovations for interventional procedures: the paradigmatic case of central venous access. Minerva Anestesiol 2020;86:121-3. DOI: 10.23736/S0375-9393.19.14070-9.

Article first published online: July 4, 2019. - Manuscript accepted: June 27, 2019. - Manuscript revised: May 21, 2019. - Manuscript received: February 17, 2019

For supplementary materials, please see the HTML version of this article at www.minervamedica.it 